PROCEEDINGS OF THE

AMERICAN MATHEMATICAL SOCIETY

Volume 128, Number 1, Pages 279-286

S 0002-9939(99)04969-2

Article electronically published on June 30, 1999

\title{
INVERSE LIMITS ON $[0,1]$ USING PIECEWISE LINEAR UNIMODAL BONDING MAPS
}

\author{
W. T. INGRAM
}

(Communicated by Alan Dow)

\begin{abstract}
In this paper we investigate inverse limits on $[0,1]$ using a single bonding map chosen from a two-parameter family of piecewise linear unimodal bonding maps. This investigation focuses on the parameter values at the boundary between an hereditarily decomposable inverse limit and an inverse limit containing an indecomposable continuum.
\end{abstract}

\section{INTRODUCTION}

In a paper [2] in the volume Continua with the Houston Problem Book the author investigated inverse limits on $[0,1]$ using a single bonding map chosen from the family of tent maps. The version of the family of tent maps considered there is given by

$$
f_{m}(x)= \begin{cases}m x & \text { if } 0 \leq x \leq \frac{1}{m}, \\ -m x & \text { if } \frac{1}{m} \leq x \leq 1,\end{cases}
$$

where $1 \leq m \leq 2$. In particular, it was shown that inverse limits on $[0,1]$ using a single bonding map chosen from the family of tent maps contain indecomposable subcontinua for $1<m \leq 2$. In that same paper, we also showed that inverse limits on $[0,1]$ using a single bonding map chosen from another family of piecewise linear bonding maps contain an indecomposable continuum beyond a certain parameter value. That family, $\boldsymbol{F}$, of maps is given by

$$
f_{t}(x)= \begin{cases}2 x & \text { if } 0 \leq x \leq \frac{1}{2}, \\ 2(1-t)(1-x)+t & \text { if } \frac{1}{2} \leq x \leq 1,\end{cases}
$$

where $0 \leq t \leq 1$. For this family it was shown that for $t<\frac{1}{2}$ the inverse limit contains an indecomposable continuum. In both families, the inverse limit is the union of a topological ray $R$ and a continuum $K$ such that $K=\bar{R}-R$. The continuum $K$ is the core of the inverse limit and results from the inverse limit on the interval $\left[f_{m}(1), 1\right]$ in the case of the tent family and $\left[f_{t}(1), 1\right]$ in the case of the family $\boldsymbol{F}$ using the appropriate restriction of the bonding map. In both the case of the tent family and the core of the family $\boldsymbol{F}$, in some sense, an arc turns out to be a "harbinger" of the onset of indecomposability in the family of inverse limits. Results in this paper indicate that this is unusual. More generally, in piecewise

Received by the editors December 11, 1996 and, in revised form, March 17, 1998. 1991 Mathematics Subject Classification. Primary 54H20, 54F15, 58F03, 58 F08.

Key words and phrases. Indecomposable continuum, unimodal mapping, inverse limit. 
linear families of maps which consist of two straight line segments, the more likely "signal" of the onset of indecomposability in inverse limits using single maps chosen from the family is a pair of $\sin \frac{1}{x}$-curves. In the last section of this paper, we include a picture (Figure 2) depicting why the arc is the indicator in the two families studied in [2] while, in general, the more likely indicator is a pair of $\sin \frac{1}{x}$-curves.

Throughout this paper, by a continuum we mean a compact, connected subset of a metric space and by a mapping we mean a continuous function. A continuum is decomposable if it is the union of two of its proper subcontinua and it is indecomposable, otherwise. If $X_{1}, X_{2}, X_{3}, \cdots$ is a sequence of topological spaces and $f_{1}, f_{2}, f_{3}, \cdots$ is a sequence of mappings such that, for each $i, f_{i}: X_{i+1} \rightarrow X_{i}$, by the inverse limit of the inverse sequence $\left\{X_{i}, f_{i}\right\}$, denoted $\lim \left\{X_{i}, f_{i}\right\}$, is meant the subset of $\prod_{i>0} X_{i}$ to which the point $x$ belongs if and only if $f_{i}\left(x_{i+1}\right)=x_{i}$ for $i=1,2,3, \cdots$. It is well known that when the spaces $X_{i}$ are continua, the inverse limit is a continuum.

A mapping is monotone provided point inverses are connected. A mapping $f:[0,1] \rightarrow[0,1]$ is called unimodal provided there is a point $c, 0<c<1$, such that $f(c)$ is in $\{0,1\}$ and the maps $f \mid[0, c]$ and $f \mid[c, 1]$ are monotone. If one considers all possible unimodal surjective maps of $[0,1]$, there are four possibilities. Such a map throws 1 to 0 , fixes 0 , throws 0 to 1 or fixes 1 . These four types were identified in [1] as Type $(i), i=1,2,3,4$. For the purposes of this paper we concentrate on Types (1) and (2) (i.e., those which throw 1 to 0 and those which fix 0 ) since we are interested in inverse limits and topologically conjugate maps produce homeomorphic inverse limits. Further, when considering a map $f$ of Type (2), the inverse limit is a topological ray limiting on the inverse limit $K=\lim \{[f(1), 1], f \mid[f(1), 1]\}$. It is easy to see that $f \mid[f(1), 1]$ is conjugate to a map of Type (1) on $[0,1]$. Thus, we will, for the most part, limit our discussion to maps of Type (1) on [0,1]. It is clear that a unimodal, piecewise linear Type (1) map $g$ of $[0,1]$ which consists of only two straight line pieces is completely determined by the choice of the critical point $c(g(c)=1)$ and $g(0)$ since $g(1)=0$. Thus, in this paper we will consider Type (1) maps determined by two parameters, $b$ and $c$, where $0 \leq b \leq 1$ and $0<c<1$. Specifically, we consider the unimodal maps ${ }^{1}$,

$$
g_{b c}(x)= \begin{cases}\frac{1-b}{c} x+b & \text { if } 0 \leq x \leq c, \\ \frac{x-1}{c-1} & \text { if } c \leq x \leq 1 .\end{cases}
$$

For convenience, we let $g_{b c}(x)=1-x$ for $b=1$ and $c=0$.

\section{ArCs AND $\sin \frac{1}{x}$-CURVES}

In this section we consider inverse limits on $[0,1]$ using a single bonding map $g_{b c}$ where $b \geq c^{2}-c+1$. In case $b>c^{2}-c+1$, we show that the inverse limit is an arc while if $b=c^{2}-c+1$, the inverse limit is a pair of $\sin \frac{1}{x}$-curves intersecting at the endpoints of the two rays. The parabola $b=c^{2}-c+1$ arises where the product of the slopes of the two pieces of $g_{b c}$ is -1 .

Theorem 1. If $b>c^{2}-c+1$, then $\lim _{\longleftarrow}\left\{[0,1], g_{b c}\right\}$ is an arc.

\footnotetext{
${ }^{1}$ Added in proof: For a discussion of the dynamics of this family, see The dynamics of a family of one-dimensional maps by Susan Bassein in the Amer. Math. Monthly, 105 (1998), 118-130
} 
Proof. Consider $g_{b c}^{2}$ (i.e., $g_{b c} \circ g_{b c}$ ). Note that its graph consists of three pieces and it throws each of the intervals $[0, p]$ and $[p, 1]$ onto itself where $p$ is the fixed point for $g_{b c}$. Further, $g_{b c}$ swaps these two intervals, so $g_{b c}$ induces a homeomorphism of the inverse limit, $\lim _{\{}\left\{[0,1], g_{b c}^{2}\right\}$, onto itself which swaps the subcontinua produced on $[0, p]$ and $[p, 1]$. If $r$ denotes the solution to $g_{b c}(x)=1$ on $[p, 1]$, then absolute value of the slope of $g_{b c}^{2}$ on $[r, 1]$ is less than 1 and $g_{b c}^{2}$ throws $[r, 1]$ into itself. Thus, $\bigcap_{n>0} g_{b c}^{2 n}[r, 1]$ is a single point and, consequently, $\varliminf_{\lfloor}\left\{[r, 1], g_{b c}^{2}\right\}$ is a single point. By Bennett's Theorem [1, Theorem 1, p. 1909], $\varliminf\left\{[p, 1], g_{b c}^{2}\right\}$ is a ray limiting on that single point, so this inverse limit is an arc. Thus, $\lim _{\longleftarrow}\left\{[0,1], g_{b c}^{2}\right\}$ is the union of two arcs intersecting only at a common endpoint and is, therefore, an arc.

Theorem 2. If $b=c^{2}-c+1$ and $c \neq 0$, then $\varliminf_{\lfloor}\left\{[0,1], g_{b c}\right\}$ is the union of two $\sin \frac{1}{x}$-curves intersecting only at the endpoint of their rays.

Proof. Proceed as in the proof of Theorem 1 considering $g_{b c}^{2}$. However, on the interval $[r, 1]$, the slope of $g_{b c}^{2}$ is -1 under the hypothesis that $b=c^{2}-c+1$. Therefore, in this case, $\varliminf_{\lfloor}\left\{[r, 1], g_{b c}^{2}\right\}$ is an arc. Now, by Bennett's Theorem [1], $\varliminf^{\lim }\left\{[p, 1], g_{b c}^{2}\right\}$ is a ray limiting on that arc. It can be shown that this is homeomorphic to a $\sin \frac{1}{x}$ curve. Thus, $\lim _{\longleftarrow}\left\{[0,1], g_{b c}^{2}\right\}$ is the union of two $\sin \frac{1}{x}$-curves intersecting only at a common endpoint of their rays.

\section{MAIN THEOREM}

A quite useful theorem for determining the presence of indecomposable subcontinua in an inverse limit on intervals using a unimodal map of Type (1) is Theorem 7 of [1]. This is the principal tool we use to establish the main theorem of this paper. In using this theorem, it is helpful to note that if $g$ is a piecewise linear unimodal map of Type (1), the first fixed point for $g^{2}$ between the critical point and the end of the interval is the fixed point for $g$. We first establish a lemma which proves useful in the general analysis of the behavior of unimodal maps of Type (1). At first glance, it may seem strange that Lemma 1 is a theorem about unimodal maps of Type (3). However, if $g$ is a unimodal map of Type (1) and $p$ is the fixed point for $g$, if $g(0)>p$, then $g^{2} \mid\left[0, g^{2}(0)\right]$ is a unimodal map of Type (3).

Lemma 1. Suppose $m>1, m^{\prime}>0$ and $B>0$. If $g:[0, B] \rightarrow[0, B]$ given by $g(x)=-m x+B$ if $0 \leq x \leq \frac{B}{m}$ and $g(x)=m^{\prime}\left(x-\frac{B}{m}\right)$ if $\frac{B}{m} \leq x \leq B$, then $g^{2}(0)$ is not less than the fixed point for $g$ if and only if $m^{2} \geq 1+\frac{m}{m^{\prime}}$.

Proof. The fixed point for $g$ is $\frac{B}{m+1}$ and $g^{2}(0)=B m^{\prime}\left(\frac{m-1}{m}\right)$. It is easy to check that $\frac{B}{m+1} \geq B m^{\prime}\left(\frac{m-1}{m}\right)$ if and only if $m^{2} \geq 1+\frac{m}{m^{\prime}}$.

Lemma 2. Under the hypothesis of Lemma 1, if $g^{2}(0)$ is less than the fixed point for $g$, then the graph of $g^{2}$ consists of three line segments of slopes $-m^{\prime}, m^{2}$ and $-m m^{\prime}$, respectively, and $g^{2}$ throws $\left[0, g^{2}(0)\right]$ onto itself.

Proof. The proof is left to the reader.

Remark. Under the hypothesis of Lemma 1, the graph of $g^{2}$ consists of three line segments whenever $g(B) \leq \frac{B}{m}$ and $g^{2}$ consists of four line segments if $g(B)>\frac{B}{m}$.

Definition. Let $\phi$ be the integer valued function defined on the non-negative integers by $\phi(0)=0, \phi(1)=1$ and $\phi(n)=\phi(n-2)+2^{n-2}$ for $n>1$. 
Lemma 3. For each positive integer $i, \phi(i+1)=\phi(i)+2 \phi(i-1)$.

Proof. We proceed by induction. It is easy to verify the lemma for $i=1$. Assume the lemma is true for $i=j$. By definition, $\phi(j+2)=\phi(j)+2^{j}$ and from the inductive hypothesis it follows that $2^{j}=2 \phi(j+1)-2 \phi(j-1)$. Thus, $\phi(j+2)=\phi(j)+$ $2 \phi(j+1)-2 \phi(j-1)=\phi(j)+\phi(j+1)+[\phi(j)+2 \phi(j-1)]-2 \phi(j-1)=$ $\phi(j+1)+2 \phi(j)$.

Lemma 4. If $i$ is an odd positive integer, $\phi(i)-2 \phi(i-1)=1$ while $\phi(i)-2 \phi(i-1)=$ -1 if $i$ is even.

Proof. We proceed by induction. It is easy to verify that the lemma is true for $i=1$. Suppose it is true for $i=j$. Note that, by Lemma $3, \phi(j+1)-2 \phi(j)=$ $\phi(j)+2 \phi(j-1)-2 \phi(j)=-(\phi(j)-2 \phi(j-1))$. The lemma is an immediate consequence of this observation.

We now turn our attention back to the map $g_{b c}$. Denote the slopes of the two pieces of $g_{b c}$ by $m_{1}$ and $m_{2}$, respectively. I.e., $m_{1}=\frac{1-b}{c}$ and $m_{2}=\frac{1}{c-1}$.

Lemma 5. Suppose $b \geq c$ and $n$ is a positive integer. Suppose further, that if $n \geq 2$, then $g_{b c}^{2^{n}}(0)$ is less than the first fixed point of $g_{b c}^{2^{n-1}}$. Then the slopes of the first two pieces of $g_{b c}^{2^{n}}$ are given by $m_{1}^{\phi(n)} \cdot m_{2}^{\phi(n+1)}$ and $m_{1}^{2 \cdot \phi(n-1)} \cdot m_{2}^{2 \cdot \phi(n)}$, respectively.

Proof. We proceed by induction. The statement is easily verified for $n=1$. Assume it holds for $n=k$ and $g^{2^{k+1}}(0)$ is less than the first fixed point for $g_{b c}^{2^{k}}$. Since the slopes of the first two pieces of $g^{2^{k}}$ are given by $m_{1}^{\phi(k)} \cdot m_{2}^{\phi(k+1)}$ and $m_{1}^{2 \cdot \phi(k-1)}$. $m_{2}^{2 \cdot \phi(k)}$, respectively, by Lemma 2 , the slopes of the first two pieces of $g^{2^{k+1}}$ are given by $m_{1}^{\phi(k)+2 \phi(k-1)} \cdot m_{2}^{\phi(k+1)+2 \phi(k)}$ and $m_{1}^{2 \cdot \phi(k)} \cdot m_{2}^{2 \cdot \phi(k+1)}$, respectively. The proof is now an immediate consequence of Lemma 3.

Theorem 3. If $b<c^{2}-c+1$, then $\varliminf_{\lfloor}\left\{[0,1], g_{b c}\right\}$ contains an indecomposable continuum.

Proof. As stated in the first paragraph of this section, our objective in this argument is to use [1, Theorem 7, p. 1912]. Noting that the fixed point for $g_{b c}$ is $\frac{1}{2-c}$, in the case that $b<\frac{1}{2-c}$, by that theorem the inverse limit is indecomposable. If $b=\frac{1}{2-c}$, the inverse limit is the union of two indecomposable continua.

We turn to the case that $\frac{1}{2-c}<b<c^{2}-c+1$. We will show that we can use Theorem 7 of [1] on $g_{b c}^{2^{n}}$ on a small interval containing 0 for a proper choice of $n$. Let $m_{1}=\frac{1-b}{c}$ and $m_{2}=\frac{1}{c-1}$. Notice that $m_{1} \cdot m_{2}=-1$ if and only if $b=c^{2}-c+1$ and so our hypothesis implies that $\left|m_{1} \cdot m_{2}\right|>1$. Also, note that $\left|m_{2}\right|>1$.

Assume that, for each positive integer $n, g_{b c}^{2^{n+1}}(0)$ is less than the first fixed point for $g_{b c}^{2^{n}}$. Then by Lemma 5 , for each $n$, the slopes of the first two pieces of $g_{b c}^{2^{n}}$ are given by $m_{1}^{\phi(n)} \cdot m_{2}^{\phi(n+1)}$ and $m_{1}^{2 \cdot \phi(n-1)} \cdot m_{2}^{2 \cdot \phi(n)}$, respectively. Thus by Lemma 1 , for odd values of $n,\left(m_{1}^{\phi(n)} \cdot m_{2}^{\phi(n+1)}\right)^{2}<1+\left|\frac{m_{1}}{m_{2}}\right|$ and for even values of $n,\left(m_{1}^{\phi(n)} \cdot m_{2}^{\phi(n+1)}\right)^{2}<1+\left|\frac{m_{2}}{m_{1}}\right|$. However, $\left(m_{1}^{\phi(n)} \cdot m_{2}^{\phi(n+1)}\right)^{2}=\left(m_{1} \cdot m_{2}\right)^{2 \phi(n)}$. $m_{2}^{2(\phi(n+1)-\phi(n))}$. Since $\left|m_{1} \cdot m_{2}\right|>1$ and $\left|m_{2}\right|>1$ and both $\phi(n)$ and $\phi(n+1)-\phi(n)$ increase without bound, this involves a contradiction. 


\section{The TENT FAMiLY}

As stated in the introduction, in [2], we showed that for the tent family, $\left\{f_{m} \mid\right.$ $1 \leq m \leq 2\}$, the inverse limit contains an indecomposable continuum if $m>1$. In particular, the core (i.e., the inverse limit on $\left[f_{m}(1), 1\right]$ using the restriction of the bonding map $f_{m}$ to that interval) of the inverse limit contains an indecomposable continuum. In the family $\boldsymbol{F}$, we showed the core of the inverse limit contains an indecomposable continuum for $t<\frac{1}{2}$. In this section, we show that these results follow directly from Theorem 3 .

Theorem 4. The core of the inverse limit using a single bonding map chosen from the tent family contains an indecomposable continuum for $1<m \leq 2$.

Proof. The restriction of $f_{m}$ to the interval $\left[f_{m}(1), 1\right]$ is topologically conjugate to the map $g_{b c}$ for $b=\frac{2 c-1}{c-1}$ where $c=\frac{1}{m}$. The set of pairs $(c, b)$ such that $b=\frac{2 c-1}{c-1}$ lies below the parabola $b=c^{2}-c+1$, thus Theorem 4 follows immediately from Theorem 3.

Theorem 5. The core of the inverse limit using a single bonding map chosen from the family $\boldsymbol{F}$ contains an indecomposable continuum for $0 \leq t<\frac{1}{2}$.

Proof. The restriction of $f_{t}$ to the interval $\left[f_{t}(1), 1\right]$ is topologically conjugate to $g_{b c}$ for $b=1-2 c$ where $b=\frac{t}{1-t}$. The set of pairs $(c, b)$ such that $b=1-2 c$ lies below the parabola $b=c^{2}-c+1$, thus Theorem 5 follows immediately from Theorem 3.

\section{Final REMARKS}

Figure 1 indicates the space of parameter pairs $(c, b)$ for which $g_{b c}$ is a map of $[0,1]$ onto itself. The space of pairs is the Cartesian product of the open interval $(0,1)$ with the closed interval $[0,1]$ together with the point $(0,1)$. On the space of parameter pairs the curves $b=c^{2}-c+1$ and $b=\frac{1}{2-c}$ have been drawn in. By Theorem 2, the parabola $b=c^{2}-c+1$ in parameter space is the set of parameter values where the inverse limit is a pair of $\sin \frac{1}{x}$-curves and below which the inverse limit contains an indecomposable continuum. Pairs above this parabola represent maps which produce arcs in the inverse limit. Along the curve $b=\frac{1}{2-c}$ the inverse limit is a pair of B-J-K continua (see [3, 2.9, page 22]) while below this curve the inverse limit is indecomposable.

Remark. The curves $b=\frac{2 c-1}{c-1}$ and $b=1-2 c$ do not intersect the parabola $b=$ $c^{2}-c+1$ for $0<c<1$ and consequently the pair of $\sin \frac{1}{x}$-curves do not appear as a signal of the onset of indecomposability in the cores of the tent family or the family $\boldsymbol{F}$. However, in one similar family, $\boldsymbol{G}=\left\{g_{t} \mid g_{t}(x)=f_{t}(1-x)\right.$ for $x$ in $[0,1]$ and $0 \leq t \leq 1\}$, investigated by the author the inverse limit is an arc for $\frac{3}{4}<t \leq 1$, a pair of $\sin \frac{1}{x}$-curves if $t=\frac{3}{4}$ and contains an indecomposable continuum if $t<\frac{3}{4}$. If, in this parameter space, one considers a curve representing a family of maps which gives inverse limits ranging from an arc to the B-J-K continuum, then from the picture it appears "more likely" that one would find a pair of $\sin \frac{1}{x}$-curves showing up in the inverse limit and, after they show up, indecomposable subcontinua would be present in the inverse limit.

Figure 2 shows Figure 1 with the curves representing the parameter values for which the maps $g_{b c}$ produce the cores of the tent family $\left(b=\frac{2 c-1}{c-1}\right)$, the cores of 


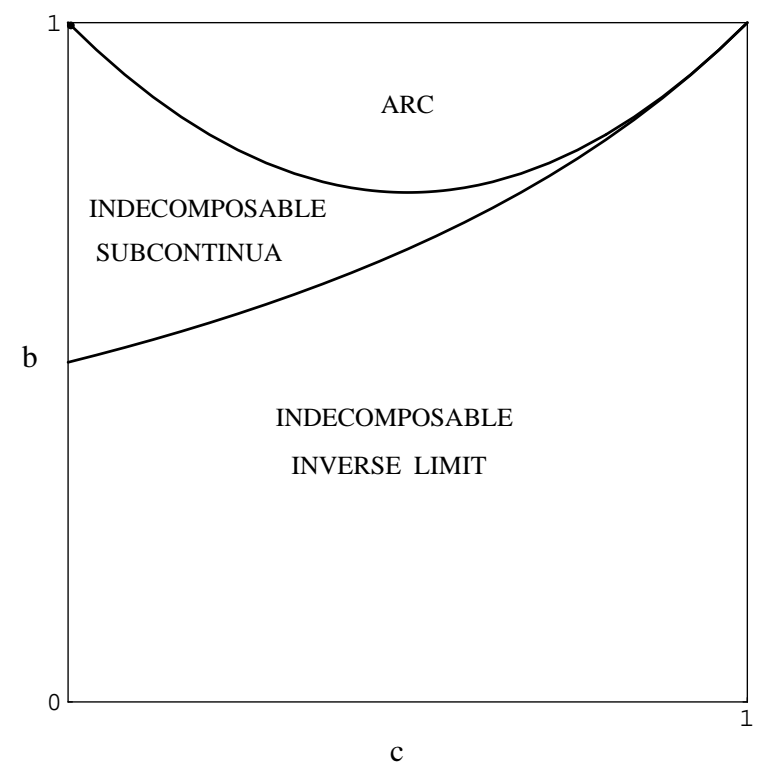

FigURE 1

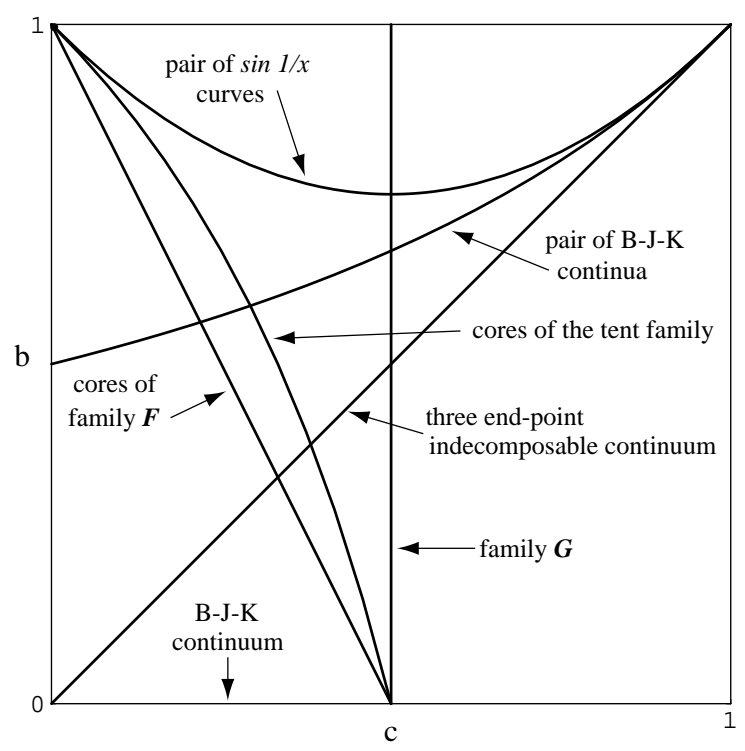

FiguRE 2

the family $\boldsymbol{F}(b=1-2 c)$ along with the family $\boldsymbol{G}\left(c=\frac{1}{2}\right)$ included. In addition, Figure 2 includes the line $b=c$ which is the set of parameter values for which map $g_{b c}$ has a periodic point of period three with 0 and 1 in the orbit. This inverse limit is the classical three endpoint, indecomposable chainable continuum. See, e.g., [3, pp.7-9]. 


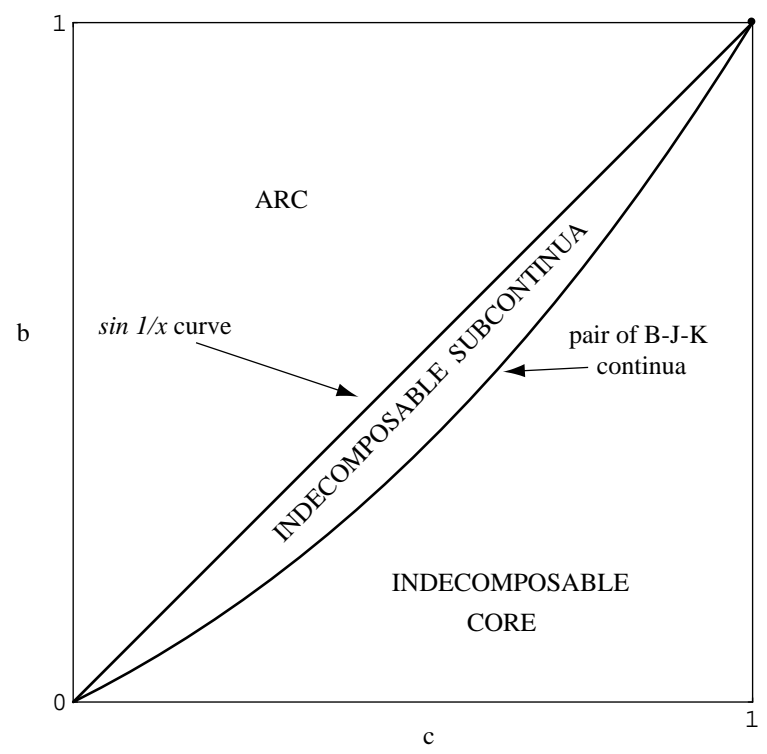

Figure 3

As Figure 1 does for the family $g_{b c}$, Figure 3 illustrates in the space of parameter pairs $\{(c, b) \mid 0<c<1,0 \leq b \leq 1\} \cup\{(1,1)\}$ the behavior for inverse limits using a single bonding map chosen from a two-parameter family of piecewise linear unimodal maps of Type (2) given by

$$
f_{b c}(x)= \begin{cases}\frac{x}{c} & \text { if } 0 \leq x \leq c, \\ \frac{b-1}{1-c}(x-1)+b & \text { if } c \leq x \leq 1\end{cases}
$$

For convenience, we let $f_{b c}(x)=x$ for $b=1$ and $c=1$. Here, if $b>c$, the inverse limit is an arc while for $b=c$ the inverse limit is a $\sin \frac{1}{x}$-curve. If $b<c$, the inverse limit contains an indecomposable continuum in its core. If $b<\frac{c^{2}-c+2+(c-1) \sqrt{c^{2}+4}}{2}$, the core is indecomposable.

Question. Suppose $(c, b)$ is a parameter pair with $0 \leq b \leq 1$ and $0<c<1$ and let

$$
C_{b c}=\left\{(x, y) \mid 0<x<1 \text { and } 0 \leq y \leq 1 \text { and } \varliminf_{\longleftarrow}^{\lim }\left\{[0,1], g_{b c}\right\}\right.
$$

be homeomorphic to $\left.\varliminf_{\lfloor}\left\{[0,1], g_{y x}\right\}\right\}$. Is $C_{b c}$ connected?

Question. Suppose $\varliminf_{\mathfrak{m}}\left\{[0,1], g_{b c}\right\}$ is homeomorphic to $\varliminf_{\lfloor}\left\{[0,1], g_{y x}\right\}$. Are $g_{b c}$ and $g_{y x}$ topologically conjugate?

One final remark. In considering inverse limits on intervals using a single piecewise linear unimodal map of Type (1) whose graph consists of two straight line intervals, the inverse limit contains an indecomposable continuum if and only if the angle between the pieces of the graph is acute. The inverse limit is an arc if and only if the angle is obtuse. The inverse limit is a pair of $\sin \frac{1}{x}$-curves if and only if the angle is a right angle. 


\section{REFERENCES}

[1] W. T. Ingram, Periodicity and indecomposablility, Proc. Amer. Math. Soc. 123 (1995), 19071916. MR 95j:58087

[2] W. T. Ingram, Inverse limits on [0,1] using tent maps and certain other piecewise linear bonding maps, Continua with the Houston Problem Book (H. Cook, W. T. Ingram, K. Kuperberg, A. Lelek and P. Minc, eds.), Marcel Dekker, New York, 1995. MR 96a:54053

[3] Sam B. Nadler, Continuum Theory, Marcel Dekker, New York, 1992. MR 93m:54002

Department of Mathematics and Statistics, University of Missouri-Rolla, Rolla, Missouri 65401

E-mail address: INGRAM@UMR.EDU 